\title{
Use of the Terms "Schizophrenia" and "Schizophrenic" in the South Korean News Media: A Content Analysis of Newspapers and News Programs in the Last 10 Years
}

\author{
Jun-Hyun Park, Young-Min Choi ${ }^{\bowtie}$, Bongseog Kim, Dong-Woo Lee and Min-Sook Gim \\ Department of Psychiatry, Sanggye Paik Hospital, College of Medicine, Inje University, Seoul, Korea
}

Objective In this study, we explored the meaning attributed to the words "jungshinbunyeolbyung" (schizophrenia) and "jungshinbunyeol" (schizophrenic) in South Korean newspapers and news programs in the last 10 years.

Methods We screened the websites of three national newspapers and the broadcasts of three nationwide television news programs from January 1, 2001, to December 31, 2010. We classified a total of 490 articles and 257 news segments by category and quantitatively and qualitatively analyzed them. The articles and news segments were assigned to one of the following categories based on their use of the term "schizophrenia": 1) negative, 2) neutral or positive, 3) incidental, and 4) metaphorical.

Results The negative viewpoint accounted for 349 incidences (46.7\%), while the neutral and positive viewpoints included 225 incidences (30.1\%). Incidental uses accounted for 95 incidences (12.7\%), and metaphorical uses accounted for 78 incidences (10.4\%). The majority of the negative uses focused on violence or dangers posed by patients (137 mentions, $37.8 \%$ ), while the metaphorical uses mainly focused on the idea of splitting (51 mentions, or 65\%).

Conclusion This study showed that the South Korean news media do not provide balanced information about schizophrenia to the public. This study also showed that no significant move has been made toward a more positive use of the term since a previous study was conducted on the subject. Although the term schizophrenia has given way to "attunement disorder," it will be difficult to establish the new term as the standard if the South Korean media continue to use the term "schizophrenic symptom." Even though the term has been changed, guidelines are necessary to encourage the mass media to provide balanced articles and reduce prejudice.

Psychiatry Investig 2012;9:17-24

Key Words Schizophrenia, Media, Stigma, Attunement disorder, Guideline.

\section{INTRODUCTION}

The media is the public's primary source of information on mental illness, ${ }^{1-3}$ and the attitude of the mass media is an important determinant of stigmatization. ${ }^{4}$ Many researchers have highlighted the role of the media in contributing to the stigma experienced by people with mental illnesses. ${ }^{5} \mathrm{~A}$ handful of studies found an association between negative media portrayals and negative attitudes toward people with mental

Received: July 9, 2011 Revised: October 28, 2011

Accepted: November 15, 2011 Available online: January 25, 2012

$\triangle$ Correspondence: Young-Min Choi, MD, PhD

Department of Psychiatry, Sanggye Paik Hospital, College of Medicine, Inje University, 761-1 Sanggye 7-dong, Nowon-gu, Seoul 139-707, Korea Tel: +82-2-950-1084, Fax: +82-2-936-8069, E-mail: ymchoi@paik.ac.kr

(c) This is an Open Access article distributed under the terms of the Creative Commons Attribution Non-Commercial License (http://creativecommons.org/licenses/bync/3.0) which permits unrestricted non-commercial use, distribution, and reproduction in any medium, provided the original work is properly cited. illnesses. ${ }^{6}$ Concerns have also been raised internationally about the role of the media in influencing public opinion and, by implication, social policy on mental health issues. In particular, concerns have been raised that an "excessive" focus by the news media on violence associated with people with mental illnesses may reinforce preexisting stereotypes, thereby causing public concern to escalate. This, in turn, could lead to the adoption of policies that place an unnecessarily high priority on public safety. ${ }^{7}$ Even balanced coverage of crimes by people with mental illnesses reinforces public perceptions that these people are violent and pose a risk to public safety. This problem is heightened when the coverage is lurid, sensationalized, or incorrect. ${ }^{8}$ Stigmatizing references to schizophrenia deter sufferers from seeking treatment and diminish the effectiveness of treatment. ${ }^{9}$ Furthermore, metaphorical references to an illness can conjure up negative, disheartening associations, ${ }^{10}$ and when these associations become commonly 
accepted, they contribute to social rejection and degradation of the well-being of persons who suffer from these illnesses. ${ }^{11}$ These stigmas, and negative views of schizophrenia in general, are enhanced by the mass media. ${ }^{12}$ Research has identified misleading and stigmatizing popular beliefs about schizophrenia, but little is known about media images that correspond to these beliefs. ${ }^{13}$

Furthermore, the former Korean term for schizophrenia, jungshinbunyeolbyung, has itself had a prejudicial effect. Unlike the Greek root of the word schizophrenia, the literal translation of the Korean term from the Chinese characters explicitly invokes the literal meaning of these characters to the general public in Korea. ${ }^{14}$ As a result, the South Korean public conceives of the disease as a frightening state in which a person's integrity is destroyed. ${ }^{14}$ Various efforts have been made to reduce these prejudices. On February 11, 2011, the Korean Society of Schizophrenia Research declared the new Korean term for schizophrenia to be johyeounbyung or "attunement disorder." ${ }^{14}$ With the change to the new, less-stigmatized name, it is an opportune time to investigate the past usage of the old term in an effort to help reduce the stigma surrounding schizophrenia. Ten years ago, a study analyzed Korean newspaper articles on psychosis. ${ }^{12}$ The study found that the term schizophrenia was used in a negative sense, 59.3\% of the time; in a neutral or a positive sense, $28.8 \%$ of the time; and with no specific bias, $11.9 \%$ of the time. However, the study analyzed themes and patterns of the use of several different terms, including jungshinbunyeolbyung, "psychosis," "mental illness," and "mental derangement." Therefore, it is hard to know exactly how the term jungshinbunyeolbyung was used by the media. Moreover, the previous study only searched for the terms in two newspapers. According to an announcement by the Korea Press Foundation, when South Koreans establish opinions or positions about public issues, the television is the most influential source of information. ${ }^{15}$ Therefore, when we consider the impact of the media on the public, it is important that we analyze TV news. Furthermore, during the last 10 years, many efforts have been made to reduce the stigma of schizophrenia. The Korean Neuropsychiatric Association designated April 4 as Mental Health Day and has held various events to reduce the stigma surrounding mental illness. ${ }^{16}$ The Korea Family Association for Mental Health has been running a mental illness recognition-improvement program and monitoring the media's treatment of mental illness. ${ }^{17}$ Further, the number of journalists who specialize in medical issues has increased during the last 10 years.

In this study, we explored the meanings attributed to the words jungshinbunyeolbyung (schizophrenia) and jungshinbunyeol (schizophrenic) in South Korean newspapers and news programs in the last 10 years.

\section{METHODS}

\section{Data}

We screened the websites of three national newspapers (Chosun-ilbo, Jungang-ilbo, and Donga-ilbo) and the broadcasts of three nationwide television news programs (KBS, MBC, and SBS) from January 1, 2001, to December 31, 2010. According to the results of the Korean Audience Survey 2010, ${ }^{15}$ these six media entities were the most influential and most viewed. We scanned all the newspaper articles and TV news broadcasts from each of these media entities for mentions of the term jungshinbunyeol, which is found in the term jungshinbunyeolbyung and can be used as an adjective meaning "schizophrenic." In order to ensure that we have not missed any article, we double-checked our list by simultaneously searching the Korea Press Foundation's integrated service and each media outlet's website. We classified a total of 490 articles and 257 news segments into four categories and analyzed them quantitatively and qualitatively. Of these, 275 articles and news segments that were not easy to classify were captured as image files using an image-capture program ${ }^{18}$ so that the raw article data could be shared with other writers.

\section{Measures}

To classify each article objectively and systematically, we assigned it to a category based on a review of articles, work in previous studies, ${ }^{12,19}$ and the survey methodology presented by Wimmer et al. ${ }^{20}$

The writers of each article were classified as journalists or non-journalists (e.g., editorialists, cultural critics, doctors, and professors), and the topics of the articles and news segments were classified as accident news, society, health, culture, domestic politics, international, economics and business, or opinions and editorials (Table 2). We then determined whether the word jungshinbunyeol (henceforth, schizophrenia) had been used 1) negatively, 2) neutrally or positively, 3) incidentally, or 4) metaphorically.

\section{Negative viewpoint}

When the term was used negatively, it was stigmatized in a way that is medically inappropriate. The characteristics of this stigmatization were determined by referencing a previous study, ${ }^{12}$ stigmas frequently encountered in clinical practice, and the 10 stigmas about mental illness identified by the South Korean Ministry of Health and Welfare. ${ }^{21}$

\section{Neutral or positive viewpoint}

When the term was used in the context of an objective, medically appropriate description of schizophrenia, we decided the viewpoint was neutral. Although the articles dealt with 
adverse events, we considered that the term was used in a neutral sense in 10 cases. In such cases, the journalists explored either the course of the disease or whether the patient was treated, and so they did not intend to directly associate the schizophrenic patients with adverse events. When the term was used in the context of favorable facts regarding persons with schizophrenia that oppose the stigma, we decided the viewpoint was positive.

\section{Incidental use}

When the disease or patient was not the main focus of the article but was mentioned in passing (e.g., price cuts for schizophrenia medicine or references to schizophrenia as a research field), we decided the term was used incidentally.

\section{Metaphorical use}

When the term was used figuratively, not literally (e.g., "their policy was very schizophrenic"), we considered it to be used metaphorically.

The above categories and individual classifications were decided through discussions among the researchers with reference to a previous study. ${ }^{12}$

\section{Analysis}

After the incidences has been categorized, we calculated the frequency and percentage of each item in each category. The three viewpoints regarding schizophrenia (negative, neutral, and positive) and the incidental and metaphorical uses of the term were analyzed qualitatively. All statistical measures were calculated using MedCalc version 11.6.2 The significance of the differences in proportions was determined using a Pearson chi-square test.

\section{RESULTS}

\section{Classification by category}

We considered all articles and news segments for analysis, with the exception of material that was duplicated in later editions of the same newspaper or broadcast. Table 1 shows the number of articles and news segments that were analyzed from each media outlet.

Some form of the blanket term "schizophrenia" was used in 747 articles and news segments. Of these, 637 (85.3\%) contained the noun form "schizophrenia" and 110 (14.7\%) contained the adjectival form "schizophrenic." A total of 440 newspaper articles $(89.8 \%)$ were written by journalists and 50

Table 1. Distribution of the use the term "schizophrenia" according to the media source in which it appeared

\begin{tabular}{|c|c|c|c|c|c|c|c|c|c|c|c|c|c|c|c|c|}
\hline & \multicolumn{8}{|c|}{ Broadcasting stations } & \multicolumn{8}{|c|}{ Newspapers } \\
\hline & \multicolumn{2}{|c|}{$\mathrm{KBS}^{\dagger}$} & \multicolumn{2}{|c|}{$\mathrm{MBC}^{\dagger}$} & \multicolumn{2}{|c|}{$\mathrm{SBS}^{\dagger}$} & \multicolumn{2}{|c|}{ Total§ } & \multicolumn{2}{|c|}{ ChoSun $\ddagger$} & \multicolumn{2}{|c|}{ JungAng ${ }^{\ddagger}$} & \multicolumn{2}{|c|}{ DongA $\ddagger$} & \multicolumn{2}{|c|}{ Total§ } \\
\hline & $\mathrm{N}$ & $\%$ & $\mathrm{~N}$ & $\%$ & $\mathrm{~N}$ & $\%$ & $\mathrm{~N}$ & $\%$ & $\mathrm{~N}$ & $\%$ & $\mathrm{~N}$ & $\%$ & $\mathrm{~N}$ & $\%$ & $\mathrm{~N}$ & $\%$ \\
\hline Negative & 63 & 60.0 & 52 & 67.5 & 50 & 66.7 & 165 & 64.0 & 61 & 36.7 & 56 & 28.3 & 66 & 52.4 & 183 & 37.3 \\
\hline Neutral* & 19 & 17.9 & 10 & 13.0 & 12 & 16.0 & 41 & 15.9 & 32 & 19.3 & 61 & 30.8 & 18 & 14.3 & 111 & 22.7 \\
\hline Positive* & 6 & 5.7 & 1 & 1.3 & 8 & 10.7 & 15 & 5.8 & 12 & 7.2 & 35 & 17.7 & 11 & 8.7 & 58 & 11.8 \\
\hline Metaphorical & 2 & 1.9 & 1 & 1.3 & 1 & 1.3 & 4 & 1.6 & 37 & 22.3 & 15 & 7.6 & 22 & 17.5 & 74 & 15.1 \\
\hline Incidental & 15 & 14.2 & 13 & 16.9 & 4 & 5.3 & 32 & 12.4 & 24 & 14.5 & 31 & 15.7 & 9 & 7.1 & 64 & 13.1 \\
\hline Total & 105 & 100 & 77 & 100 & 75 & 100 & 257 & 100 & 166 & 100 & 198 & 100 & 126 & 100 & 490 & 100 \\
\hline
\end{tabular}

${ }^{*}$ in comparing the media outlets, the neutral and positive viewpoints were combined, ${ }^{+} \chi^{2}=11.862, \mathrm{df}=6, \mathrm{p}=0.065,{ }^{\ddagger} \chi^{2}=29.756, \mathrm{df}=6, \mathrm{p}<0.0001$, $\mp \chi^{2}=20.16, \mathrm{df}=3, \mathrm{p}<0.0001$. KBS: Korean Broadcasting System, MBC: Munwha Broadcasting Corporation, SBS: Seoul Broadcasting System

Table 2. Comparison of viewpoints according to the type of newspaper articles and TV news segments in which they appeared

\begin{tabular}{|c|c|c|c|c|c|c|c|c|c|c|}
\hline \multirow{2}{*}{ Type } & \multicolumn{2}{|c|}{ Negative } & \multicolumn{2}{|c|}{ Neutral and positive } & \multicolumn{2}{|c|}{ Metaphorical } & \multicolumn{2}{|c|}{ Incidental } & \multicolumn{2}{|c|}{ Total } \\
\hline & $\mathrm{N}$ & $\%$ & $\mathrm{~N}$ & $\%$ & $\mathrm{~N}$ & $\%$ & $\mathrm{~N}$ & $\%$ & $\mathrm{~N}$ & $\%$ \\
\hline Society & 149 & 42.7 & 66 & 29.3 & 9 & 11.5 & 37 & 38.9 & 261 & 34.9 \\
\hline Health & 20 & 5.7 & 118 & 52.4 & 0 & 0.0 & 26 & 27.4 & 164 & 22.0 \\
\hline Accident news & 108 & 30.9 & 7 & 3.1 & 0 & 0.0 & 2 & 2.1 & 117 & 15.7 \\
\hline Culture & 40 & 11.5 & 21 & 9.3 & 41 & 52.6 & 13 & 13.7 & 115 & 15.4 \\
\hline International & 23 & 6.6 & 7 & 3.1 & 8 & 10.3 & 8 & 8.4 & 46 & 6.2 \\
\hline Economics and business & 2 & 0.6 & 1 & 0.4 & 11 & 14.1 & 8 & 8.4 & 22 & 2.9 \\
\hline Opinions and editorials & 7 & 2.0 & 5 & 2.2 & 7 & 9.0 & 1 & 1.1 & 20 & 2.7 \\
\hline Politics & 0 & 0.0 & 0 & 0.0 & 2 & 2.6 & 0 & 0.0 & 2 & 0.3 \\
\hline Total & 349 & 100 & 225 & 100 & 78 & 100 & 95 & 100 & 747 & 100 \\
\hline
\end{tabular}


newspaper articles (10.2\%), by non-journalists.

Table 2 summarizes the results in terms of the kind of article or news segment the term was used in. The negative viewpoint accounted for 349 incidences (46.7\%), while the neutral and positive viewpoints accounted for 225 incidences (30.1\%). We found 95 incidental uses of the term $(12.7 \%)$ and 78 metaphorical uses (10.4\%).

Articles and news segments associated with military service accounted for 58 mentions (7.8\%). Twenty of these were articles and news segments dealing with exemption from military service because of schizophrenia, while 38 dealt with the onset of schizophrenia during mandatory military service.

\section{Classification by period}

We compared two periods, 2000-2004 and 2005-2010, to determine whether the term schizophrenia was used differently over time (Figure 1). Negative mentions increased from 147 to 202 in the latter period, neutral or positive mentions decreased from 119 to 105 , and metaphorical uses decreased from 45 to 33 .

\section{Content analysis of negative viewpoints}

Negative viewpoints were most frequently found in society news, with 149 mentions (42.7\%). The negative viewpoints can be grouped into six categories, discussed below (Table 3).

\section{Schizophrenic patients are dangerous or violent or may commit a crime}

The majority of negative viewpoints focused on violence or the dangers posed by patients (137 mentions, 37.8\%). These articles referred to individuals with schizophrenia using stigmatizing descriptors such as "causeless assault," "brutally stabbing," and "cruelly." Even though the writers did not have access to a certain diagnosis, when there were psychotic symptoms, the articles used the expression "schizophrenic symptoms," which can evoke a relation to schizophrenic patients. Of the 117 mentions in accident news, 60 (51.3\%) checked whether the schizophrenic patient had visited the hospital and only 28 (23.9\%) reported their course of treatment.

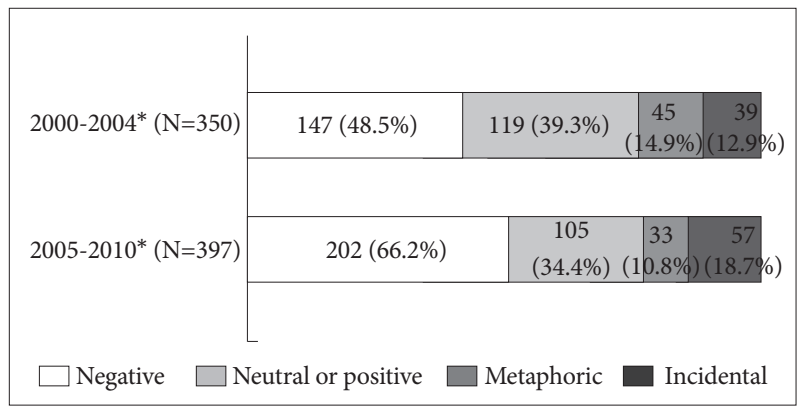

Figure 1. Comparison of the use of the term "schizophrenia" form 2000-2004 and 2005-2010. ${ }^{*} \chi^{2}=3.811, d f=3, p=0.283$.

\section{Particular events cause schizophrenia}

In 82 cases (23.5\%), the negative viewpoints were about the causes of schizophrenia. The writers portrayed a particular event as being directly related to the cause of the disease, without balanced bio-psycho-social consideration. In 82 cases, 25 of the stories $(30.4 \%)$ were related to mandatory military service. Some stories also attributed the disease to specific drugs, personal character, masturbation, high heels, and other features.

\section{Schizophrenic patients are bizarre or grotesque}

In 63 cases (18.1\%), the negative viewpoints were about a patient's bizarreness, regardless of the disease's state or the patient's course of treatment. Some references to individuals with schizophrenia used terms like "mysticism." By using "suddenly" as a descriptor, the media placed too much emphasis on the possible unpredictability of patients. In some movie and book reviews, schizophrenia was depicted in the form of a man acting extremely strange.

\section{Schizophrenia is incurable}

In 36 cases (10.3\%), negative viewpoints were expressed through descriptions of schizophrenia as incurable. When discussing the limitations of drug therapy, schizophrenia was presented as an archetypally incurable disease. In these negative cases, patients eventually committed suicide or became mentally disabled as a result of their incurable schizophrenia.

\section{Schizophrenic patients cannot function well in society}

In 28 cases (8\%), the articles or news segments described schizophrenic patients as behaving inappropriately at normal social functions. These stories argued that schizophrenic patients should not, for example, be permitted to drive or have a job and that they should be categorically distinguished from patients with other mental disorders. One article described the feeling of being threatened by schizophrenic patients cook-

Table 3. Negative viewpoints describing schizophrenia

\begin{tabular}{lc}
\hline \multicolumn{1}{c}{ Items } & Frequency $(\%)^{*}$ \\
\hline $\begin{array}{l}\text { Patients are dangerous or violent } \\
\text { or may commit a crime }\end{array}$ & $137(37.8)$ \\
Particular events cause it & $82(23.5)$ \\
Patients are bizarre or grotesque & $63(18.1)$ \\
It is incurable & $36(10.3)$ \\
Patients cannot function well in society & $28(8.0)$ \\
Patients should be institutionalized & $22(6.3)$ \\
\hline
\end{tabular}

*the numbers in parentheses indicate the percentage of the total negative newspaper articles and TV news segments $(\mathrm{N}=349)$. When two or more themes were expressed in one article, they were coded separately. Therefore, the total frequency and percentage exceed 349 and $100 \%$ respectively 
Table 4. Positive viewpoints describing schizophrenia

\begin{tabular}{lc}
\hline \multicolumn{1}{c}{ Items } & \multicolumn{1}{c}{ Frequency (\%)* } \\
\hline $\begin{array}{l}\text { Encouragement and suggestions } \\
\text { for reducing stigma }\end{array}$ & $47(64.4)$ \\
Information about the treatment \\
of schizophrenia
\end{tabular}

ing with knives. Further, we found statements about how the limitations of people with the serious illness of schizophrenia should be acknowledged.

\section{Schizophrenic patients should be institutionalized}

In 22 cases (6.3\%), the negative viewpoints were about the deinstitutionalization of schizophrenic patients. Within accident news, one article highlighted patients who had recently been discharged from the hospital. Another article argued that homeless people with mental illnesses should be isolated from society.

\section{Content analysis of neutral and positive viewpoints}

Neutral and positive viewpoints most frequently appeared in health stories, with 118 mentions (52.4\%). Of the 73 positive viewpoints, most (47 mentions, 64.4\%) were related to active encouragement and suggestions for improving the social perception of schizophrenia and reducing its stigma. Information about how to treat schizophrenia accounted for 41 mentions (56.2\%), and medical attitudes regarding the causes of schizophrenia accounted for 22 mentions (30.1\%). Seventeen articles and news segments contained two or more positive viewpoints (Table 4).

\section{Content analysis of metaphorical uses}

Metaphorical uses were not limited to any particular area of reporting but were more common in culture stories (41 mentions, 52.6\%) (e.g., "a schizophrenic character," "a diary that is almost schizophrenic," and "a schizophrenic promotion").

In metaphorical uses, the meaning was mainly related to the idea of splitting (51 mentions, 65\%) (e.g., "ideological struggles make our society schizophrenic" and "the publishing world is in a schizophrenic state"). The next most common meaning was "contradictory" (20 mentions, 26\%) (e.g. "protectionism and expansion, which are contradictory in a schizophrenic way" and "it was a schizophrenic state, speaking left wing and acting right wing"). In seven cases (9\%), the term meant "odd"

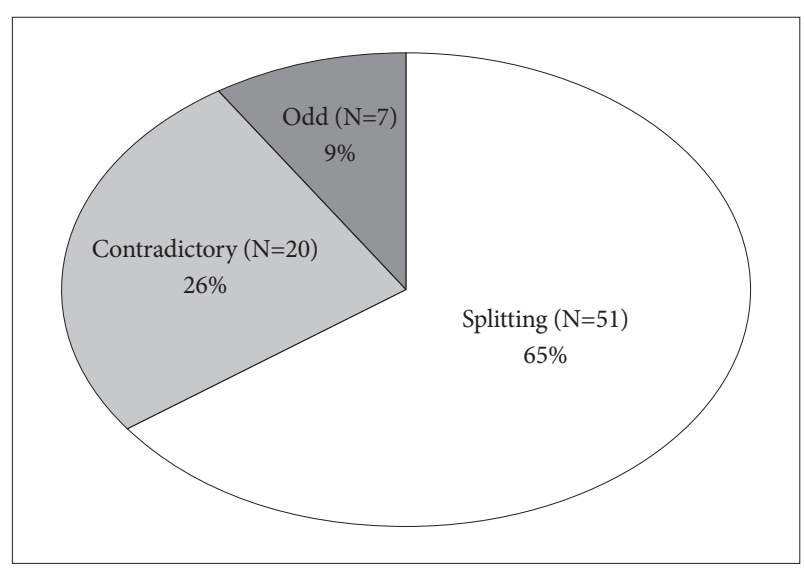

Figure 2. The meaning of "schizophrenia" in metaphorical use.

(e.g., "his actions, which we can't understand, were schizophrenic") (Figure 2).

Of the 78 cases of metaphorical use, 26 (33.3\%) were written by non-journalists. In the articles and news segments written by journalists, only 17 (21.8\%) used schizophrenia metaphors. Thirty-four other cases (43.6\%) quoted interviewees or other people using the term metaphorically.

\section{DISCUSSION}

By observing the changing use of the word "schizophrenia," we can understand the public's views on the disease. Accordingly, by considering that metaphorical usages (78 mentions; $10.4 \%$ of all usages) have a negative and stigmatizing effect, they could be classified as a negative usage. Thus, nearly $57.1 \%$ $(10.4 \%+46.7 \%)$ of articles and news segments that used the term did so negatively, compared to the $30.1 \%$ that had a neutral or positive view of the term. This hints that balanced information is not provided to the public.

Our comparison of the previous research (1998-2000) with this research (2000-2010) showed there was no significant difference in the negative or positive ratios of the use of the term schizophrenia in newspapers $\left(X^{2}=1.455, \mathrm{df}=2, \mathrm{p}=0.483\right)$. However, although this result is not significant, according to the results of research by the institute responsible for the current research, in the past 10 years (divided into two five-year periods, 2000-2004 and 2005-2010), the ratio of negative meanings has increased, while that of positive and neutral meanings has decreased. Various efforts have been made to reduce prejudice toward schizophrenia during the last decade. The above result, however, shows that no significant move has been made toward a more positive use of the term. However, one should also consider whether the use of the word for schizophrenic patients has been corrected from other misused terms, such as "psychosis" and "mental derangement."

Perhaps, the term schizophrenia itself has a negative bias. 
In 1977, Susan Sontag described how illnesses whose etiologies are considered mysterious are prone to be used as metaphors. ${ }^{23}$ It is already known that the names of feared diseases are usually employed with a metaphorical emphasis. In the nineteenth century, for example, metaphors about tuberculosis were very common, while in the twentieth century, these have largely been replaced with metaphors about cancer. ${ }^{4}$ Over the course of the two periods mentioned above, the use of schizophrenia as a metaphor declined. Moreover, compared to other countries, metaphorical uses in Korea made up a smaller proportion of total uses. Research from the United States, ${ }^{13}$ Germany, ${ }^{24}$ and Switzerland ${ }^{25}$ reported that $28 \%, 58 \%$, and $31 \%$, respectively, of the uses of the word schizophrenia in newspapers were metaphorical. The lower percentage in Korea might be the result of efforts by medical scientists, who have worked hard over the past decade to reduce prejudice toward schizophrenia. However, it may also be that in the Korean culture, the term jungshinbunyeol is regarded as too harsh to be casually used as a metaphor.

Negative views were most frequently related to violence and crime $(37.8 \%)$. This result is not significantly different from previous studies. ${ }^{12}$ Indirect and direct mentions of schizophrenia as the cause of accidents were frequent. Provocative comments were also used to describe accidents and crimes caused by schizophrenic patients. Even in cases where there had been no diagnosis of schizophrenia, the words of families, lawyers, police, and suspects were used without filtering to support arguments that the people who committed the crimes were schizophrenic. This suggests that the reason for this phenomenon may be efforts to find the cause of crimes that cannot be accepted by society. Schizophrenic patients become scapegoats during this process. In 117 accident news stories, only $60(51.3 \%)$ checked whether the patient had ever visited the hospital. The other 57 (48.7\%) mentioned that the suspect was schizophrenic without having proper medical evidence. The course of treatment or exact status of the disease was reported in only 28 cases $(23.9 \%)$. Checking how these patients have been treated and determining their status at the time of the accident would help reduce prejudice toward schizophrenic patients in general.

The abusive use of the term "schizophrenic symptoms" (jungshinbunyeol jeongsae) also contributes to the negative understanding of schizophrenia. The term "schizophrenic symptoms" was also used for psychotic symptoms caused by depression with psychotic features, post-operation delirium, and the side effects of drugs. If a person's symptoms are not caused by psychotic episodes stemming from schizophrenia, the term "psychotic symptom" should be used instead of "schizophrenic symptom." Although the term schizophrenia has given way to attunement disorder, it will continue to be diffi- cult to establish the new term as the standard if the South Korean media continue to use the term schizophrenic symptom.

Of all the articles and news segments that used the word schizophrenia, $7.8 \%$ were related to military service. This high rate could be due to South Korea's draft. The media needs to pay special attention when they release news about military exemptions related to schizophrenia because the news can give the impression that the diagnosis of schizophrenia is unscientific and arbitrary. Some news articles reported that the cases of schizophrenia emerging within the military were directly related to specific violent incidents or accidents. Other articles and news segments discussed a violent incident that had occurred in the military. It had recently been reviewed by the courts, and the victim was given national meritorious acclaim. Although this is a positive sign from the point of view of enhancing human rights, it is also dangerous, as it neglects the biological-weakness factor in schizophrenia and places too much emphasis on the psychological and social elements of the disease.

The use of the term differed between newspapers and TV news segments $(\mathrm{p}<0.0001)$. This may be because TV programs about health were not included in this research. The term was also used differently in each newspaper $(\mathrm{p}<0.0001)$. The mean and standard deviation of the number of negative usages in each newspaper were 61.00 and \pm 5.00 respectively, and the mean and standard deviation of the number of neutral and positive usages in each newspaper were 19.33 and \pm 13.57 respectively. Thus, the differences among newspapers was greater for the positive and neutral usages than for the negative usages $\left(\chi^{2}=27.175, \mathrm{df}=2, \mathrm{p}<0.0001\right)$.

It is possible that a certain number of events related to schizophrenia are likely to be described negatively. This implies that the differences in the use of the term in each newspaper are not due to differences in the negative views of each media outlet, but to differences in the positive views, which encourage efforts to overcome prejudice. Neutral and positive views occurred most frequently in health stories; 47 articles and news segments were related to active encouragement and suggestions for improving the social perception of schizophrenia and reducing its stigma. This may be the result of various efforts made by psychiatry associations and society at large, or it could be related to the increase in the number of reporters who specialize in medical areas. Having neutral and positive views does not mean having blindly positive views of schizophrenia. For instance, when schizophrenia occurred after an accident, some articles mentioned that the accident could have triggered the disease in someone who was already predisposed to it. Some articles stated that children who are born to older parents have a higher risk of schizophrenia, but on the same day, another article reported the same fact while noting 
that it could simply be a case of correlation, not causation.

\section{Limitations of the research}

It was possible to search all the newspaper sections, but our ability to search TV programs, except for on-air news, was limited, so only newspaper articles and on-air news programs were used for this research. Thus, we could not include information about how the term schizophrenia was used in dramas, comedies, and other programs. Nowadays, people do not just watch the news, but they also comment on it or write blogs about it. Analyzing the public's reactions to reports related to schizophrenia is also an important reflection of the public's views. Just because the word is used negatively, we cannot say that the reporter has a negative view of it; the general level of understanding in society also has an effect. Moreover, the situation is also affected by the source itself and the interviewee. In some cases, it was difficult to distinguish between the negative and neutral viewpoints, and we found that there was a significant overlap between the two viewpoints. An untreated patient who exhibits an acute psychotic state is actually dangerous and bizarre.

\section{Conclusion}

The National Alliance on Mental Illness monitors the mass media (newspapers, TV, and movies) and publishes sharp rebuttals when it finds prejudice or improper characterizations of schizophrenia. As a result, it has improved the quality of treatment for schizophrenic patients. In Korea, the Korea Family Association also sends letters to the mass media showing the results of their monitoring. To enhance the effectiveness of these approaches, psychiatry associations and other related organizations need to provide guidelines. Hence, we propose the following.

In order to ensure that a report is accurate, it should contain the exact and latest information on schizophrenia. In addition, owing to the media stories about schizophrenia, people often desire more information about the disorder. Therefore, it will be helpful to develop an accurate report that provides symptoms, causes, treatments, prognoses, associations with violence, etc., and to provide it to journalists and broadcasters. Additionally, it will be better to include information about the helpline and contact details. Further, inappropriate terminology and misuse of the term schizophrenia make it difficult to convey its proper meaning. Thus, including a glossary of terms associated with schizophrenia in the guidelines would help to reduce the incorrect use of the terms. While reporting accident-related news, the guidelines should encourage journalists and broadcasters to check whether the person had visited a hospital, been diagnosed by a doctor, taken medicine, etc.

Currently, formal legal processes are under way to change the Korean term for schizophrenia to attunement disorder. It is expected that this will diminish some of the stigma surrounding schizophrenia. Perhaps integrating efforts could now exert a synergy effect. Regardless, guidelines will be necessary even after the term is changed to encourage the mass media to release balanced articles and reduce prejudice.

\section{Acknowledgments}

The main findings of this paper were presented by poster at the 2 nd Asian Congress on Schizophrenia Research on February 11, 2011.

\section{REFERENCES}

1. Borinstein AB. Public attitudes toward persons with mental illness. Health Affairs 1992;3:186-196.

2. Philo G. Media Representation of Mental Health/Illness: Audience Reception Study. Glasgow: Glasgow University Media Group; 1994.

3. Kalafatelis E, Dowden A. Public Knowledge of, and Attitudes to, Mental Health and Illness. Wellington: Business Research Centre for Ministry of Health; 1997.

4. Boke O, Aker S, Alptekin Aker A, Sarisoy G, Sahin AR. Schizophrenia in Turkish newspapers: retrospective scanning study. Soc Psychiatry Psychiatr Epidemiol 2007;42:457-461.

5. Ferriman A. The stigma of schizophrenia. BMJ 2000;320:522.

6. Dietrich S, Heider D, Matschinger H, Angermeyer MC. Influence of newspaper reporting on adolescents' attitudes toward people with mental illness. Soc Psychiatry Psychiatr Epidemiol 2006;41:318-322.

7. Paterson B. Newspaper representations of mental illness and the impact of the reporting of "events" on social policy: the "framing" of Isabel Schwarz and Jonathan Zito. J Psychiatr Ment Health Nurs 2006;13: 294-300.

8. Heginbotham C. UK mental health policy can alter the stigma of mental illness. Lancet 1998;352:1052-1053.

9. Warner R. Combating the stigma of schizophrenia. Epidemiol Psichiatr Soc 2001;10:12-17.

10. Link BG, Cullen FT, Frank J, Wozniak JF. The social rejection of former mental patients: understanding why labels matter. Am J Sociol 1987; 92:1461-1500

11. Perlick DA, Rosenheck RA, Clarkin JF, Sirey JA, Salahi J, Struening EL, et al. Stigma as a barrier to recovery: adverse effects of perceived stigma on social adaptation of persons diagnosed with bipolar affective disorder. Psychiatr Serv 2001;52:1627-1632.

12. Kim SW, Yoon JS, Lee MS, Lee HY. The analysis of newspaper-articles on psychosis. J Korean Neuropsychiatr Assoc 2000;39:838-848.

13. Duckworth K, Halpern JH, Schutt RK, Gillespie C. Use of schizophrenia as a metaphor in US newspapers. Psychiatr Serv 2003;54:1402-1404.

14. Lee YS, Kwon JS. JoHyeonByung (Attunement Disorder); The birth of a new Korean term for schizophrenia. The 2nd Asian Congress on Schizophrenia Research. Seoul, Korea, Feb 2011.

15. Korea Press Foundation. The Result of Korean Audience Survey 2010. Seoul: Korea Press Foundation; 2011.

16. Medical News Today. Why did we make April 4 Mental Health Day? Available at: http://suicide.blutouch.net/bbs/board.php?bo_table= press_room\&wr_id=139\&page=2. Accessed June 25, 2011.

17. Korea Family Association for Mental Health Corp. Mental Illness Recognition Improvement Program: Media Monitoring. Seoul: Korea Family Association for Mental Health Corp; 2009.

18. Ancamera (image-capture program). Available at: http://www.ancamera.com/. Accessed June 25, 2011.

19. Williams M, Taylor J. Mental illness: media perpetuation of stigma. Contemp Nurse 1995;4:41-46.

20. Wimmer RD, Dominick JR. Mass Media Research: An Introduction, 9th Ed. Belmont, CA: Wadsworth Publishing Company; 2011. 
21. Korean Neuropsychiatric Association. Changing the 10 Stigmas of Mental Illness (Newsletter of Korean Neuropsychiatric Association). Seoul: Korean Neuropsychiatric Association, 2000; 7.

22. MedCalc (statistical software). Available at: http://www.medcalc.org/. Accessed June 25, 2011.

23. Sontag S. Illness as Metaphor. New York: Struss and Giroux; 1996.
24. Hoffmann-Richter U, Forrer F, Finzen A. Schizophrenia in the German national paper Frankfurter Allgemeine Zeitung-a didactic play. Psychiatr Prax 2003;30:4-7.

25. Hoffmann-Richter U, Alder B, Hinselmann V, Finzen A. [Schizophrenia in the "New Zurich Newspaper." A media analysis]. Psychiatr Prax 1998; 25:14-18. 